\title{
PREDICTION OF PLASTIC DEGRADING MICROBES
}

\author{
${ }^{1 *}$ Hemalatha N, ${ }^{1}$ Akhil Wilson, 1 Akhil T \\ ${ }^{1}$ St Aloysius College of Management and Information Technology
}

Abstract: Plastic pollution is one of the challenging problems in the environment. But a life without plastic we cannot imagine. This paper deals with the prediction of plastic degrading microbes using Machine Learning. Here we have used Decision Tree, Random Forest, Support vector Machine and $K$ Nearest Neighbor algorithms in order to predict the plastic degrading microbes. Among the four classifiers, Random Forest model gave the best accuracy of $99.1 \%$.

Keywords: Plastic, Microbes, Degradation, Random Forest

\section{Introduction}

Plastic is nothing but a polymeric material. Plastic pollution has become one of the most stressing environmental issue, as rapidly increasing production of disposable plastic products overwhelms the world's ability to deal with them. We cannot think of a life without plastic. Plastics revolutionized medicine with life-saving devices, made space travel possible, lightened cars, jets saved fuel and pollution, also saved lives with helmets, incubators, equipment's for clean drinking water but on the other side it makes lot of environmental pollution. Practically it is difficult to avoid plastic completely from our daily life. The only solution to control the plastic pollution is degrading the plastic products rather than throwing it into the surroundings. Using the proper management, we can reduce the pollutions in the environment than what plastic creates.

Streptococcus, Micrococcus, Staphylococcus, Moraxella, Psedomonas these are the some of the plastic degrading microbes found in Indian mangrove soil. This outcome was a result of Japanese scientists in the year 2016 in which they found that a bacterium can easily break the plastic polyethylene terephthalate (PET). Further, they also found another bacterium called Ideonella sakaiensia obtained from the genus Ideonella and from the family Comamonadaceae which can break the plastic polyethylene terephthalate (PET). Once the bacterium acts, PET gets broken down into two i.e., ethylene glycol and DMT which can be used to create other materials. In this paper we have worked on developing a computational prediction tool with regard to plastic degrading protein sequences. Also have developed a database which consists of protein sequences collected from previous works which can degrade plastics. This research paper has been distributed like this: part 2 represents the different materials and also the methodology used to carry out this work. Then followed by the results and discussions in part 3 and Paper is concluded in part 4.

\section{Methodology}

This section describes the dataset used, different algorithms and features used for generating the computational tool.

\subsection{Dataset}


Plastic degrading protein sequences belonging to the alkB and CYP153 genes were cumulated from databases such as NCBI and UniprotKB. Around nine thousand positive protein sequences and six thousand negative sequences were obtained. For training data, we used the ratio 60:40 for positive and negative sequences.

\subsection{Features Used}

Six different features namely amino acid counts, dipeptide counts, amino acid ratio, hydrophobicity, hydrophilicity, acidity and basicity from microbial protein sequences were used for developing predicton. Features are explained in below sections.

\subsubsection{Amino acid count}

In the amino acid count, we took the count of all the 20 amino acids in each microbial protein sequence making the dimension size 20 (Table 1). Equation used is

$$
\text { A. } A \text { Count }=\mathrm{N}\left(\mathrm{a}_{\mathrm{i}}\right) \text { where }[\mathrm{i}=1 \text { to } 20]
$$

\subsubsection{Amino acid ratio}

Here, count of each amino acid in a sequence was divided by the length of protein sequence. For this feature, feature dimension was 20 (Table 1). Equation used is

$$
\text { A. A Ratio }=\frac{\mathrm{N}\left(\mathrm{a}_{\mathrm{i}}\right)}{\text { length }} \text { of the protein sequence }
$$

\subsubsection{Dipeptide count}

In the dipeptide count, count of the occurrence of all dipeptide in the protein sequence was taken and dimension was of size 400 (Table 1).

$$
\text { Dipep Count }=N\left(a_{i} a_{j}\right) \text { where }[i, j=1 \text { to } 20]
$$

\subsubsection{Physiochemical properties}

In this feature, hydrophobicity, hydrophilicity, acidity and basicity were considered (Table 1). Hydrophobicity had 9 amino acids and hence dimension of 6 whereas hydrophilicity six, acidity two and basicity had a dimension of three. 
Table 1. Details of the amino acids contained in different features

\begin{tabular}{|c|c|}
\hline Features & Amino Acids \\
\hline Amino Acid count & 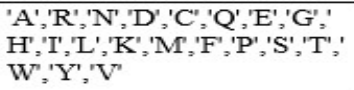 \\
\hline dipeptide & 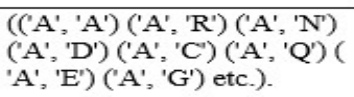 \\
\hline hydrophobicity & 'A','G','I','L','M,',F','P', ,'V', 'W \\
\hline hydrophilicity & 'S', 'T",'C','N',',Q','Y' \\
\hline Acidic & 'D','E' \\
\hline basicity & 'K','H','R' \\
\hline
\end{tabular}

\subsection{Data Pre-processing}

Handling of the missing values is very important during the preprocessing as many machine learning algorithms do not support the missing data. The missing data can impact the performance of the model which is done through creating bias in the dataset. Hence, we can say that this bias can create a lack of relatability and trustworthiness in the dataset.

\subsection{Algorithms used}

In order to classify the plastic degrading microbes, we used the supervised machine learning techniques such as classification algorithms. Following subsections explains each classifier in detail.

2.4.1 Decision trees: Decision Tree algorithm is a classification algorithm which has nodes and leaves. Nodes are split based on certain conditions and outcomes are obtained on the leaves. This is one of the most commonly using simplest classification algorithm.

2.4.2 Random Forest: It is a classification algorithm in which it generates multiple decision trees based on the dataset. Each of the decision trees predicts the output and then finally it combines the outputs by voting.

2.4.3 Support Vector Machine: This classifier creates a hyperplane on the dataset and then classifies the data points into classes.

2.4.4 KNN (K-Nearest Neighbour): It known as a lazy-Learner algorithm. This algorithms classifies the input values based on the similarity.

\subsection{Performance measures}

For measuring the performance of the computational prediction tool measures used were accuracy, confusion matrix, ROC-AUC and heat map which are described below.

\subsubsection{Accuracy}

Accuracy can be defined as the ratio of number of the correct predictions to the total number of input values . 


$$
\text { Accuracy }=\frac{(\text { True Positive }+ \text { True Negative })}{(\text { True Positive }+ \text { True Negative }+ \text { False positive }+ \text { False Negative) }}
$$

\subsubsection{Confusion matrix}

Confusion Matrix which gives the output in the matrix form and explains the whole evaluation measures of the model which we created.

Table2: Measures of confusion matrix.

\begin{tabular}{|l|l|l|}
\hline Measures & Predicted Class & Actual Class \\
\hline True Positive & Yes & Yes \\
\hline True Negative & No & No \\
\hline False Positive & Yes & No \\
\hline False Negative & No & Yes \\
\hline
\end{tabular}

F1 Score we can define as a measurement of the models accuracy or harmonic mean of precision and the recall. This ranges from 0 to 1 . It tells you that among the data how many instances it predicts correctly. The precision and recall can be calculated by using following equations.

$$
\begin{array}{cc}
\text { Precision }= & \mathrm{TP} \\
\cline { 2 - 2 } \text { Recall }= & \mathrm{TP}+\mathrm{FP} \\
\cline { 2 - 2 } & \mathrm{TP} \\
\mathrm{TP}+\mathrm{FN}
\end{array}
$$

\subsubsection{ROC-AUC}

AUC can be expanded as Area Under Curve. It is one of the metrics used for evaluation. As the value of the AUC rises the model performance for classification also rises. Before studying the AUC, we need to understand following measurements.

The True Positive Rate(TPR) we can defined as TP/ (FN+TP). It measures among all actual positive samples how many are correctly classified.

True Negative Rate(TNR) can be defined as TN / (FP+TN).

False Positive Rate(FPR) can be defined as FP / (FP+TN). It measures among all actual negative samples how many are incorrectly classified.

\subsubsection{Heat map}

The heat map is nothing but a data visualization technique which helps to detect the correlation between the features.

\subsection{Scikit learn}

Scikit learn is a python library which gives many supervised and unsupervised learning algorithms. For this research work we have used NumPy, pandas and matplotlib from this library, 


\section{Results and Discussions}

This section discusses the results obtained on pre-processing the dataset and then working the features of the dataset with four different machine learning classifiers.

\subsection{Data pre-processing}

In the present work, since twenty amino acids were compulsorily involved in all the plastic degrading microbes the chances of existence of the null values were nil. Using python environment, this was confirmed. Dataset used were also confirmed for skewness and existence of outliers. Details of all the three pre-processing are available in Table 3.

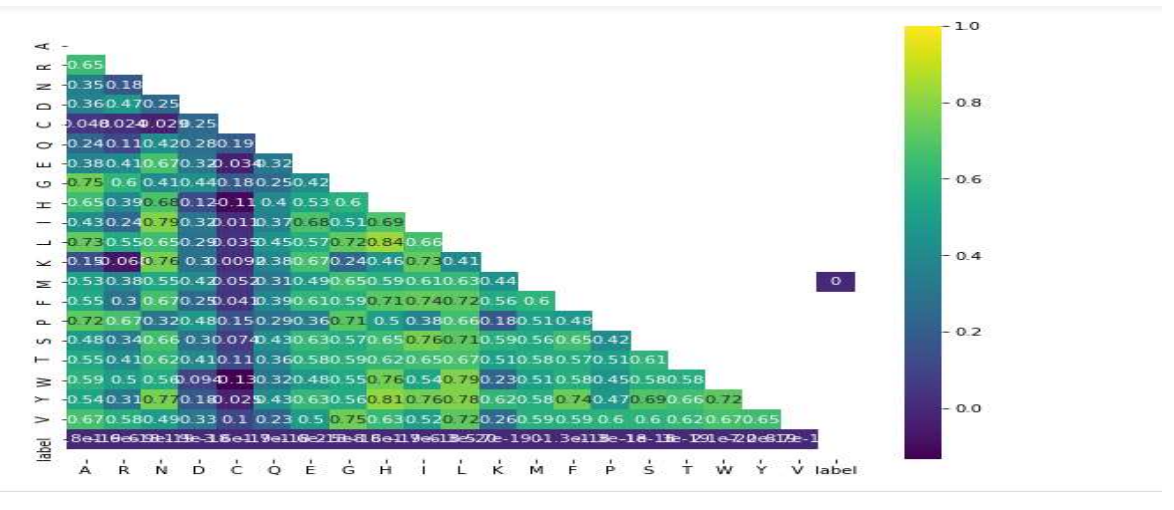

Fig 1: Heatmap

Table 3: Table showing results of missing values, skewness and existence of outlier

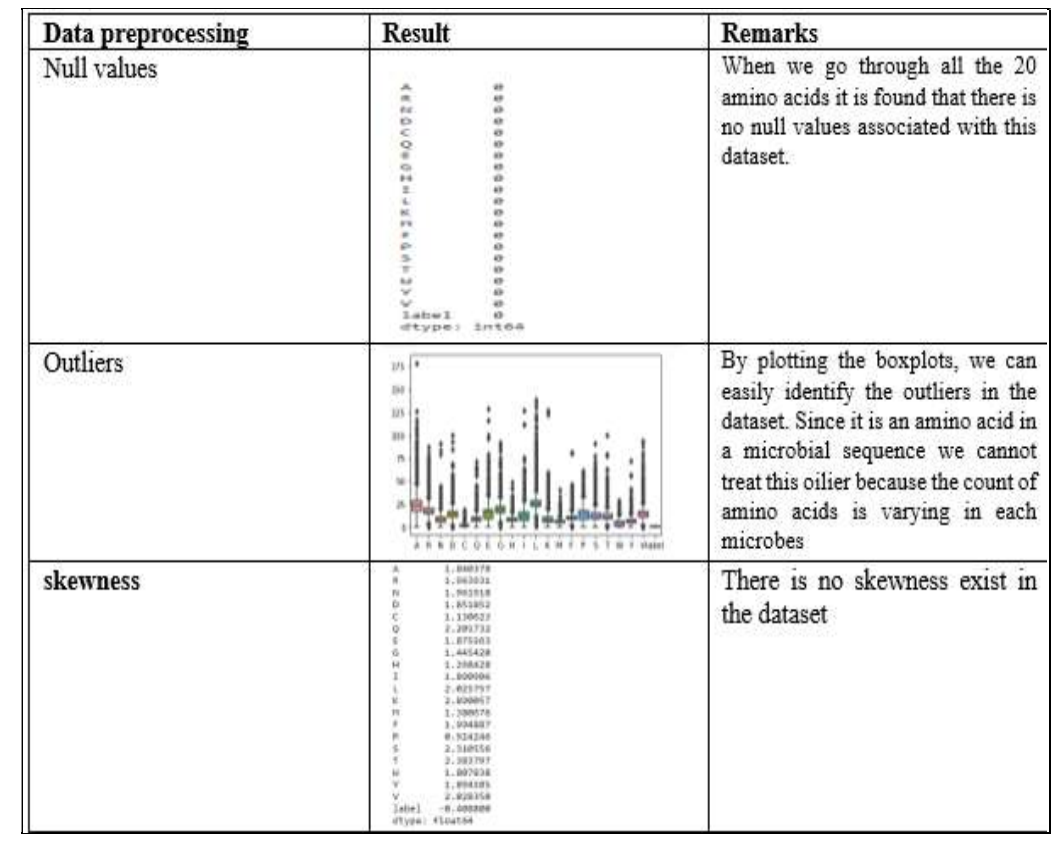




\subsection{Algorithm results}

During experimentation of six features with four classifiers namely decision tree, Random Forest, SVM and KNN model obtained different accuracies which are listed in Table 4. From Table 5 it could be concluded that Random forest and KNN obtained best accuracy for the feature amino acid count. Diagrammatic representation is shown in Figure 2 and 3.

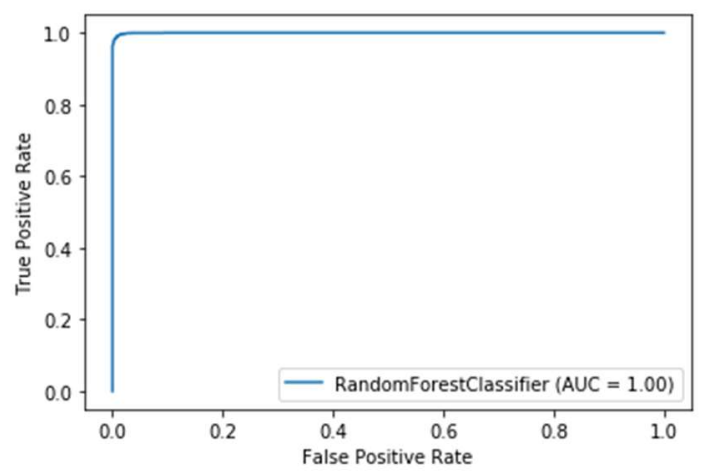

Fig 2: ROC-AUC curve of Random Forest for Amino acid count

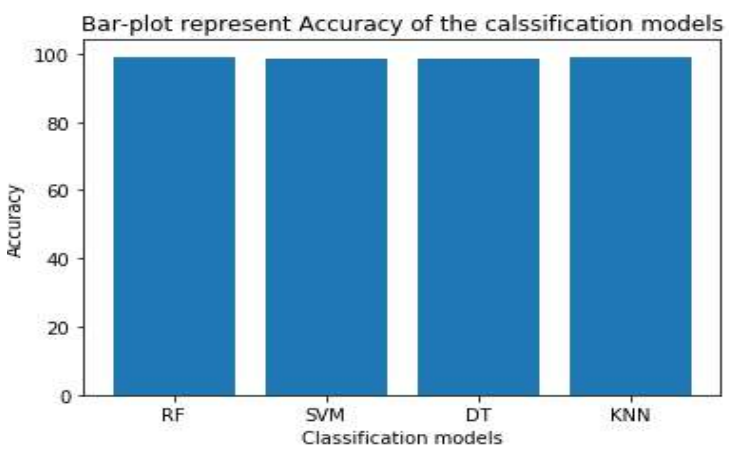

Fig 3: Bar diagram indicating the accuracies of 4 classifiers for amino acid count

Table 4: Accuracies of different algorithms

\begin{tabular}{|l|l|l|l|l|l|l|l|}
\hline Model & $\begin{array}{l}\text { Amino } \\
\text { Acid Co } \\
\text { unt }\end{array}$ & $\begin{array}{l}\text { Dipeptid } \\
\text { e }\end{array}$ & $\begin{array}{l}\text { hydroph } \\
\text { obic }\end{array}$ & $\begin{array}{l}\text { Hydroph } \\
\text { ilic }\end{array}$ & Acidic & Basic & $\begin{array}{l}\text { Amino } \\
\text { Acid Rat } \\
\text { io }\end{array}$ \\
\hline RF & 99.1 & 12.088 & 98.433 & 93.733 & 89.2 & 84.866 & 98.766 \\
\hline SVM & 98.7 & 36.55 & 96.133 & 88.7 & 89.0333 & 59.9 & 97.5 \\
\hline DT & 98.33 & 12.08 & 97.166 & 90.5 & 89.16 & 83.7 & 98.3 \\
\hline KNN & 99.1 & 26.88 & 98.266 & 90.733 & 89.0 & 83.666 & 99.2 \\
\hline
\end{tabular}


Table 5: Accuracies in Amino acid count

\begin{tabular}{|l|l|}
\hline Model & accuracy \\
\hline RF & $\begin{array}{l}99.13333333333 \\
333\end{array}$ \\
\hline SVM & 98.7 \\
\hline DT & $\begin{array}{l}98.33333333333 \\
333\end{array}$ \\
\hline KNN & 99.1 \\
\hline
\end{tabular}

\section{Conclusion}

In the present world to sustain without plastic is unimaginable but to overcome such situation only way out is to use microbes which can degrade the used plastic. In this work, we have attempted to develop a computational prediction tool which can classify a microbial protein if it is biodegradable or not. Four machine learning algorithms were used for this purpose along with six different features of $p$ roteins. Out of the six features, it was found that the Amino acid count gave an accuracy of $99.1 \%$ with Random Forest and KNN classifiers. In the future work, we plan to develop a web server where we can host the prediction tool.

\section{Acknowledgement}

This work is a part of the major research project funded by St. Aloysius Management.

\section{References}

[1] Caruso, Gabriella. "Plastic degrading microorganisms as a tool for bioremediation of plastic contamination in aquatic environments." J Pollut Eff Cont 3.3 (2015): 1-2.

[2] Raziyafathima, M., P. K. Praseetha, and R. S. Rimal Isaac. "Microbial degradation of plastic waste: a review." Chemical and Biological Sciences 4 (2016): 231-42.

[3] Kale, Swapnil Kisanrao, et al. "Microbial degradation of plastic: a review." Journal of Biochemical Technology 6.2 (2015): 952-961.

[4] Vignesh, R., et al. "Screening of plastic degrading microbes from various dumped soil samples." Int Res $J$ Eng Tech 3.4 (2016): 2493-2498.

[5] Kale, Swapnil Kisanrao, et al. "Microbial degradation of plastic: a review." Journal of Biochemical Technology 6.2 (2015): 952-961.

[6] Asmita, Kamble, Tanwar Shubhamsingh, and Shanbhag Tejashree. "Isolation of plastic degrading micro-organisms from soil samples collected at various locations in Mumbai, India." International Research Journal of Environment Sciences 4.3 (2015): 77-85.

[7] Bano, Kulsoom, et al. "Microbial enzymatic degradation of biodegradable plastics." Current pharmaceutical biotechnology 18.5 (2017): 429-440.

[8] Devi, Rajendran Sangeetha, et al. "The role of microbes in plastic degradation." Environ. Waste Manage 341 (2016). 
[9] Aghavi, Navid, et al. "Degradation of plastic waste using stimulated and naturally occurring microbial strains." Chemosphere 263 (2021): 127975.

[10] Jaiswal, Shweta, Babita Sharma, and Pratyoosh Shukla. "Integrated approaches in microbial degradation of plastics." Environmental Technology \& Innovation 17 (2020): 100567.

[11] Holmes, Geoffrey, Andrew Donkin, and Ian H. Witten. "Weka: A machine learning workbench." Proceedings of ANZIIS'94-Australian New Zealnd Intelligent Information Systems Conference. IEEE, 1994.

[12] Kotsiantis, Sotiris B., I. Zaharakis, and P. Pintelas. "Supervised machine learning: A review of classification techniques." Emerging artificial intelligence applications in computer engineering 160.1 (2007): 3-24.

[13] Sen, Pratap Chandra, Mahimarnab Hajra, and Mitadru Ghosh. "Supervised classification algorithms in machine learning: A survey and review." Emerging technology in modelling and graphics. Springer, Singapore, 2020. 99-111.

[14] Singh, Amanpreet, Narina Thakur, and Aakanksha Sharma. "A review of supervised machine learning algorithms." 2016 3rd International Conference on Computing for Sustainable Global Development (INDIACom). Ieee, 2016. 